\title{
ORAL HEALTH-RELATED QUALITY OF LIFE AND ITS ASSOCIATED FACTORS IN ELDERLY POPULATION
}

\author{
Shazia Liaquat ${ }^{1}$, Aliya Khan', Imaad khan', Aleena Malik², Waqar Azeem³ \\ ${ }^{1}$ Department of preventive and community Dentistry, Khyber College of Dentistry \\ ${ }^{2}$ House officer, Ayub teaching hospital Abbottabad \\ ${ }^{3}$ House officer, Sardar Begum hospital, Peshawr - Pakistan
}

\begin{abstract}
Objectives: This study was aimed to evaluate oral health-related quality of life and its associated factors in the elderly.

Material and Methods: A cross-sectional study was done at the medical Out Patient Department of Khyber Teaching Hospital Peshawar by using a non-probability consecutive sampling method of 411 elderly patients. All the patients had an oral examination, followed by an interview through a validated questionnaire having demographics and oral health-related quality of life (OHRQoL) was measured using OHIP-14 having Likert scale, with higher scores indicating poor oral health (lower OHRQOL) and vice versa. Results: The mean OHIP-14 score (range 0-56) was 62.35 \pm 10.94 which is much higher than the threshold for poor OHRQoL 'poor OH' $\geq 11.0$ (SD \pm 6.9 ). OHRQoL was found good in the participants having good oral Hygiene (51.74) and who were more qualified. Females had relatively good OHRQoL (61.4). The mean OHIP score was higher in the participants belonging to rural areas (67.1) as compared to urban areas.

Conclusion: This study found poor OHRQoL in the elderly population in Peshawar. Participants who had good oral hygiene \& who were qualified had good OHRQoL. OHRQoL was found poor in rural areas participants; it was relatively good in females as compared to males.
\end{abstract}

Keywords: Elderly population, Oral Health-Related Quality of Life, Oral Hygiene, Oral Health Impact Profile.

This article may be cited as: Liaquat S, Khan A, khan I, Malik A, Azeem W. Oral Health-Related Quality of Life and its Associated Factors in Elderly Population. J Med Sci 2021 July;29(3):91-95

\section{INTRODUCTION}

The expansion of the definition of health by WHO led to the concept of wellbeing and wellbeing is measured by improvement in quality of life $e^{1,2}$.

As the oral health-related quality of life is a multi-dimensional thought, which deals with dental health and its impact upon wellbeing, therefore, it is difficult to assess OHRQoL. Research groups have made considerable efforts to develop a tool for the measurement of the impact of impairment on life. Most of the instruments concentrate on problems in the oral cavity and some of them assess the positive effects of oral condition on OHRQoL $3,4,5$.

The oral health impact profile is the questionnaire that is mostly used for measuring Oral health-related quality of life. This Questionnaire quantifies the impact of oral issues

\section{Correspondence}

Dr. Shazia Laiquat

Associate Professor,

Department of Preventive and Community Dentistry,

Khyber College of Dentistry, Peshawar - Pakistan

Email: makhdoomshazia@yahoo.com

Cell: $+92-331-8855230$

Date received: $\quad 07-11-2020$

Date revised: $06-08-2021$

Date accepted: $08-08-2021$ and covers physical, psychological, and social dimensions of daily routine. Its original version had 49 items later on it was changed to OHIP-14, it is so named because it has 14 dominions. This shortened form of OHIP-14 had equal sensitivity to the old version which had 49 items It is divided into seven domains having two questions in each domain. These seven domains are (functional limitation, physical pain, psychological discomfort, physical disability, psychological disability, social disability, and handicap). The responses are scored on a Likert scale having five points which are from never to very often. OHIP-14 has been used in the Pakistani population by Ibrahim Warsi et al in Karachi $^{6,7}$.

There is a paucity of research in this province on the effect of oral hygiene status, education level, gender, and address on OHRQoL. The objective of the present research was to find the relation of OHRQoL with oral hygiene, qualification, gender, and address.

\section{MATERIAL AND METHODS}

This research was carried out to measure oral health and its related quality of life in the older population coming to Khyber Teaching Hospital (KTH), Peshawar. Research data was collected in the outpatients' department of the Department of medicine of Khyber Teaching hospital over 
three months using a non-probability consecutive sampling technique. All patients above the age of 60 years, visiting the medical outpatient department of the MTI KTH for seeking treatment of their clinical diseases were recruited in the study. Patients with serious clinical issues including blood disorders, physically and intellectually debilitating conditions were excluded. Research data was collected after the informed consent of the participants. Taking population proportion of $50 \%$, a margin of error of 0.05 , and power of study $80 \%$ in WHO sample size calculator the calculated sample size was 385 , while 411 participants were easily included in the study.

After the ethical approval for the research and informed written consent, the patients had oral examination using a disposable spatula and convenient light. After having the oral examination, all the participants were asked questions in the questionnaire having demographics and OHIP-14 used for measuring oral health-related quality of life.

The questionnaire of OHIP-14 is divided into seven domains with each domain having two questions. These seven domains are (functional limitation, physical pain, psychological discomfort, physical disability, psychological disability, social disability, and handicap).

The answers of the participants were scored on a Likert scale having five points which are from never to very often. The range of the OHIP-14 scale is 0 to 56 where greater scores show poor OHRQoL and lower score shows good OHRQoL. The standard value for 'good oral health is $\leq 9.33 \pm 6.5$ and for poor Oral hygiene is $\geq 11.0 \pm 6.9$. Overall Visual Oral Hygiene was defined as apparently generally visualized by the observer.

Data was entered and analysis was performed on SPSS version 21 , keeping the confidence interval at $95 \%$ and $a$ value at 0.05 . Analysis of descriptive variables was done and a t-test was performed for mean comparison OHIP score in two groups. ANOVA was used to compare the mean OHIP score between more than two groups.

\section{RESULTS}

Out of the total 411 participants, the mean age was $67.79 \pm 5.3$ with an age range from $60-80$ years of age. The mean OHIP Score (range 0-56) of this study was calculated as $62.35 \mathrm{SD} \pm 10.940$ and this is greater than the value for bad OHRQoL, which is OHIP-14 score greater than $11 \pm 6.5$, by applying one-sample t-test $t$ value was greater 91.23 p-values .000 thus showing a greater difference between the mean score of this study and standard value which is 11.

This table shows that majority were reported as "quite often" having had problems in the last one year on 10 of the 14 items (ranging from Experience of disturb sleep (41.6), avoid smiling (42.67), food getting stuck (45.3), avoid eating a certain food (47.2), feeling less confident (49.1), the experience of bad breath (51.6), experience discomfort eating (53.8), spending a lot of money on dental problems (57.9), experience shyness (59.6) and experience of ulcers (60.6). There were relatively fewer participants who reported that they "sometimes" had problems of disturbed concentration (40.1) \& avoid going out (41.6). Regarding the variable of overall visual oral hygiene applicants with good oral hygiene had a good oral health-related quality of life followed by those with fair oral hygiene and those who had poor oral hygiene had poor oral health related quality of life (Table. 4).

On account of the variable of the level of education those participants who were illiterate, had a high mean OHIP score (64), followed by primary (61.68), matric (61.58), and intermediate (59.11), while those participants who were graduates had a lesser score of 47.15 . Hence this study shows that as the level of education increases the mean OHIP score decreases and the quality of life gets better (Table. 4).

By applying the Independent Sample T-test it was found that the male participants had relatively higher OHIP score i.e. $62.7 \pm 10.36$, as compared to the female with $61.4 \pm 12.08$ with a p-value of 0.235 , thus females had a relatively good oral health-related quality of life as compared to males (Table 4) and this was statistically significant. According to the variable of address, the mean OHIP score was higher i.e. 67 in participants belonging to rural areas as compared to urban areas which were 58 , which means the oral health-related quality of life was good in the urban participant as compared to rural participants (table-4). Table 4: Distribution of visual oral hygiene, educational level, and gender with OHIP score

Table 1: Baseline Characteristics of the participants

\begin{tabular}{|c|c|c|c|c|}
\hline Baseline Characteristics & Categories & Frequency & Total Frequency & \%age \\
\hline \multirow{2}{*}{ Age in years } & $69-60$ & 250 & 411 & 60.8 \\
\cline { 2 - 4 } & $80-70$ & 161 & & 39.2 \\
\hline \multirow{2}{*}{ Gender } & Male & 256 & 411 & 62.3 \\
\cline { 2 - 4 } & Female & 155 & & 37.7 \\
\hline Address & Rural & 188 & 411 & 45.7 \\
\cline { 2 - 4 } & Urban & 223 & \\
\hline
\end{tabular}


Oral Health-Related Quality Of Life And Its Associated Factors In Elderly Population

Table 2: One-Sample Statistics

\begin{tabular}{|c|c|c|c|c|}
\hline \multicolumn{5}{|c|}{ OHIP score of the applicant } \\
\hline $\mathrm{N}$ & Mean & SD & S.E. Mean & P-Value \\
\hline 409 & 62.35 & 10.940 & 0.541 & 0.005 \\
\hline
\end{tabular}

Table 3: Description of the Answers to OHIP-14

\begin{tabular}{|c|c|c|c|c|c|c|c|c|}
\hline \multicolumn{9}{|c|}{ Distribution of OHIP items ranging from 0 (never) 1 (seldom) 2 (sometimes) 3 (quite often) 4 (very often) } \\
\hline \multicolumn{2}{|c|}{ Description of items } & \multicolumn{7}{|c|}{ Distribution of responses (\%) $n$} \\
\hline Items & Questions & 0 & 1 & 2 & 3 & 4 & Mean & S.E. \\
\hline \multicolumn{9}{|c|}{ Functional Limitations } \\
\hline $\mathrm{OH}-1$ & Chewing difficulty & $(5.4) 22$ & $(10.5) 43$ & $(25.1) 103$ & $48.9(201)$ & $10.2(42)$ & 2.48 & 0.49 \\
\hline $\mathrm{OH}-2$ & Bad breath & (7.8) 32 & (13.1) 54 & (20.4) 84 & (51.6) 212 & (7.1) 29 & 2.37 & 0.052 \\
\hline \multicolumn{9}{|c|}{ Physical Pain } \\
\hline $\mathrm{OH}-3$ & Discomfort eating & (3.2) 13 & $(10.7) 44$ & $(23.4) 96$ & $(53.8) 22$ & (19) 37 & 2.55 & 0.045 \\
\hline $\mathrm{OH}-4$ & Ulcers & (4.6) 19 & (11.7) 48 & (11.4) 47 & (60.6) 249 & (11.7) 48 & 2.63 & 0.048 \\
\hline \multicolumn{9}{|c|}{ Psychological Discomfort } \\
\hline $\mathrm{OH}-5$ & Food getting stuck & (5.4) 22 & (5.6) 23 & (32.8) 135 & (45.3) 186 & (10.9) 45 & 2.51 & 0.047 \\
\hline $\mathrm{OH}-6$ & Feeling shy & (2.2) 9 & (9.7) 40 & (17.5) 72 & (59.6) 245 & (80.9) 45 & 2.67 & 0.043 \\
\hline \multicolumn{9}{|c|}{ Physical Disability } \\
\hline $\mathrm{OH}-7$ & Avoid eating & $(5.4) 22$ & $(8.8) 36$ & (27.5) 113 & (47.2) 194 & (11.2) 46 & 2.50 & 0.049 \\
\hline $\mathrm{OH}-8$ & Avoid smiling & (7.5) 31 & $(0.7) 44$ & (28.7) 118 & (42.6) 175 & (10.5) 43 & 2.38 & 0.052 \\
\hline \multicolumn{9}{|c|}{ Psychological Disability } \\
\hline $\mathrm{OH}-9$ & Disturbed sleep & (8.3) 34 & (12.2) 50 & (27.3) 112 & (41.6) 171 & (10.5) 43 & 2.35 & 0.054 \\
\hline $\mathrm{OH}-10$ & $\begin{array}{c}\text { Disturbed concentra- } \\
\text { tion }\end{array}$ & (3.9) 16 & $(9.7) 40$ & $(40.1) 165$ & (36.0) 148 & $(10.2) 42$ & 2.39 & 0.046 \\
\hline \multicolumn{9}{|c|}{ Social Disability } \\
\hline $\mathrm{OH}-11$ & Avoid going out & (8.6) 27 & (31.6) 13 & (41.6) 171 & (10.2) 42 & (10) 44 & 1.85 & 0.051 \\
\hline $\mathrm{OH}-12$ & Less confident & (5.1) 21 & (10) 41 & (26.3) 108 & (49.1) 202 & (19.5) 39 & 2.48 & 0.048 \\
\hline \multicolumn{9}{|c|}{ Handicap } \\
\hline $\mathrm{OH}-13$ & Daily activities affected & (5.1) 21 & (10.9) 45 & (43.1) 177 & (32.4) 133 & (8.5) 35 & 2.28 & 0.047 \\
\hline $\mathrm{OH}-14$ & $\begin{array}{l}\text { Increase expenditure } \\
\text { on dental problems }\end{array}$ & (7.3) 30 & (9.0) 37 & $(15.1) 62$ & (57.9) 236 & (11.1) 46 & 2.57 & 0.052 \\
\hline
\end{tabular}

Table 4: Distribution of visual oral hygiene, educational level, and gender with OHIP score

\begin{tabular}{|c|c|c|c|c|c|}
\hline & & $\mathbf{N}$ & Mean & S.D & P-Value \\
\hline \multirow[t]{3}{*}{ Visual oral hygiene } & Good & 53 & 51.74 & 9.340 & \multirow[t]{3}{*}{$<.005$} \\
\hline & Fair & 83 & 52.81 & 8.812 & \\
\hline & Poor & 275 & 67.11 & 8.408 & \\
\hline \multirow[t]{5}{*}{ Levels of Education } & Illiterate & 183 & 64.38 & 8.471 & \multirow[t]{5}{*}{$<.005$} \\
\hline & Primary & 124 & 61.68 & 12.619 & \\
\hline & SSC & 64 & 61.58 & 13.888 & \\
\hline & HSSC & 27 & 59.11 & 5.423 & \\
\hline & Bachelors \& above & 13 & 47.15 & 5.273 & \\
\hline \multirow{2}{*}{$\begin{array}{c}\text { Gender of the } \\
\text { participants }\end{array}$} & Male & 256 & 62.74 & 10.360 & \multirow[t]{2}{*}{.235} \\
\hline & Female & 155 & 61.41 & 12.084 & \\
\hline \multirow{2}{*}{$\begin{array}{l}\text { Address of the } \\
\text { participants }\end{array}$} & Rural & 188 & 67.1 & 8.521 & \multirow[t]{2}{*}{$<.005$} \\
\hline & Urban & 223 & 58 & 8.201 & \\
\hline
\end{tabular}




\section{DISCUSSION}

The mean OHIP score in the present study was greater than $11 \pm 6.5$ which is the cut-off value for bad oral health, thus showing a greater difference between the mean score of this study and the standard value which is 11. This means that the oral health-related quality of life of the participants was found poor. Similar findings were found in the study done by Warsi et $\mathrm{al}^{7}$ where the OHIP score was $23.38 \pm 10.47$ i.e. oral health-related quality of life was also poor. The findings of Kim et al, on the other hand, contradict the findings of the present study in which the mean OHIPscore was $10.66 \pm 10.7$ which is in the range of good oral health-related quality of life ${ }^{8}$. In a study done by Young et all in 2009 in Korean elders, the median OHIP score was 7 , which was also in the range of good oral health related quality of life ${ }^{9}$.

Participants having good oral hygiene had a good oral health-related quality of life, these findings are in line with the findings of Kung Hee Lee 2013 in west Virginia those participants who had lower OHIP scores also had good oral hygiene ${ }^{10}$.

Illiterate participants had high OHIP scores i.e. poor oral health-related quality of life as compared to educated participants. The same findings were reported by Kari Elisabeth 2010 in which individuals with higher education had a good oral health-related quality of life ${ }^{11}$. The present results are in line with those of previous studies and confirm that level of education is associated with oral health-related quality of life Ekback et al ${ }^{12}$.

In our study, there was a statistically insignificant $(p$-value $=0.23$ ) association between gender and the OHIP score of the participants. These findings contradict the results of Kari Elisabeth Dahl in 2011 in which women reported poorer oral health-related quality of life as compared to the males participants ${ }^{13}$. Einarson et al also reported the same findings in their study in 2009 that females have a poor oral health-related quality of life relatively to males and likewise same findings were found in the study of Inukai et al in 2010 in which oral health-related quality of life was good in males (correlation coefficient -0.60 ) as compared to females $(-0.46)^{14,15}$. The findings of Kim et all study in 2009 are however in agreement with the results of the present study, that male participants had a higher mean OHIP score (12.2 SD 11.0) as compared to females (9.8 SD 10.5) ${ }^{8}$. Factors responsible for the better oral health of females is that they are more conscious about their health and fitness.

Participants from the urban area had a good oral health-related quality of life as compared to rural areas. These findings are similar to the results of McGrath C, John MT, Mesalu JR in which OHRQoL is found good in urban areas, which might be because rural people have much less access to the health services which lead to late treatment $16,17,18$.

\section{LIMITATION OF THE STUDY}

One limitation of our study is that it was carried out in a hospital setting which is not ideal; it however provides baseline data over which further research can be done for finding the actual Oral health-related quality of life mean value in a community setting.

\section{CONCLUSION}

According to this research Oral health-related quality of life was found poor in geriatrics and confirms that oral health also has impacts on other aspects of life. Those who had a positive attitude towards oral health had a good quality of life.

\section{REFERENCES}

1. Mohammed AJ, Ghebreyesus TA. Healthy living, well-being and the sustainable development goals. Bull World Health Organ.2018;96(9):590-590

2. Chahar P,Mohanty VR, Aswini YB. Oral health-ralated quality of life among elderly patients visiting special clinics inpublic hospital in Delhi, India: A cross sectional study. Indian J public Health 2019;63(1): 15-20. DOI: 10.4103/ijph.IJPH_316_17

3. Wong FMF, Yannies NTY, leung wk. Oral health and its Associated Factors among institutionalized Residents- A systematic review. Int J Environ. Res. Public Health 2019,16-4132pdf ; https://doi.org/10.3390/ ijerph16214132

4. Masood et al.The relationship between oral health and oral health related quality of life among elderly people in United Kingdom Dent 20017; vol.56:78-83.

5. Orti'z- Barrios,L.B., Granados- Garcia, V., Cruz-Hervert, $P$. et al. he impact of poor oral health on the oral health related quality of life (OHRQoL) in older adults: the oral health status through a latent class analysis. BMC oral Health 2019. 141.DOI. https://doi.org/10.1186/s12903019-0840-3

6. Vitti RP, Oliviera ARBde, Sinhoreti MAC, Barakat LF, Ucheonye IJ. Oral Health Impact Profile (OHIP-14) and its association with dental treatment needs of adolescents in a rural Nigerian community. Brazilian Journal of Oral Sciences 2017:15(3);2015-20

7. I Warsi et al. Oral health related quality of life in patients with upper gastrointestinal and hepatic disorders in $\mathrm{Pa}$ kistan: validation of Oral Health Impact Profile-14 in the Urdu language.BDJ.2018(4)17036.

8. Kim et al. effect of malocclusion severity on oral health related quality of life and food intake ability on Korean population Am.J of Orth.2016;149(3):384-390.

9. Young et al. chewing function impacts oral health related quality of life among institutionalized and community dwelling Korean elders. Community Dent. \&Orlal Epidemiol.2009;37(5):468-476.

10. $\mathrm{KH}$ lee et al.Cognitive function and oral health-related quality of life in older adults.Journal of American Geriatrics society.2013;61(9):1602-1607.

11. Dahl KE, Wang NJ, Skaul, Øhrn K. Oral health-related 
quality of life and associated factors in Norwegian adults. ActaOdont Scan doi:10.3109/00016357.2010.549502.

12. Stahlnacke IK, UnellL, soderfeldt B,Ekback G,Ordells. self-perceived oral health among 65 and 75 years olds in two Swedish countries.Swedish Dental Journal .2010;34(2):107-119.

13. Dahl KE, Wang NJ, Holst D, Øhrn K. Oral health-related quality of life among adults 68-77 years old in Nord-Trøndelag, Norway. Int J Dent Hygiene2011; 9:87-92.

14. Einarson S, Gerdin EW, Hugoson A. Oral health impact on quality of life in an adult Swedish population. ActaOdont Scan2009;67:85-93.

15. Inukai, M., John, M.T., Igarashi, Y. et al. Association between perceived chewing ability and oral healthrelated quality of life in partially dentate patients. Health Qual Life Outcomes 8, 118 (2010) doi:10.1186/14777525-8-118.

16. McGrath C, Comfort MB, Lo EC, Luo Y. Can third molar surgery improve quality of life?A 6-month cohort study.J Oral Maxillofac surg.2003;61:759-63.

17. John MT, LeResche L, Koepsell TD, Hujoel P, Miglioretti DL, Micheelis W. Oral health-related quality of life in Germany.Eur J Oral Sci.2003;111:483-91.

18. Masalu JR, Astrom AN. Social and behavioral correlates of oral quality of life studied among university students in Tanzania.ActaOdontol Scand.2002;60-353
CONFLICT OF INTEREST: Authors declare no conflict of interest

GRANT SUPPORT AND FINANCIAL DISCLOSURE: NIL

\section{AUTHOR'S CONTRIBUTION}

Following authors have made substantial contributions to the manuscript as under

Liaquat S: Idea and conceptualization, manuscript writing, final critical review and appraisal

Khan A: $\quad$ Planning, manuscript writing, formatting and critical review

khan I: $\quad$ Manuscript writing and critical review

Malik A: $\quad$ Data searching, manuscript writing and critical review

\section{Azeem W: $\quad$ Manuscript writing}

Authors agree to be accountable for all aspects of the work in ensuring that questions related to the accuracy or integrity of any part of the work are appropriately investigated and resolved. 